Classification

Physics Abstracts

$05.20-87.30$

\title{
Effect of synapse dilution on the memory retrieval in structured attractor neural networks
}

\author{
N. Brunel (*) \\ Laboratoire de Physique Statistique, Ecole Normale Supérieure, 24 rue Lhomond, F-75231 Paris \\ Cedex 05, France
}

(Received 22 February 1993, accepted 27 April 1993)

\begin{abstract}
We investigate a simple model of structured attractor neural network (ANN). In this network a module codes for the category of the stored information, while another group of neurons codes for the remaining information. The probability distribution of stabilities of the patterns and the prototypes of the categories are calculated, for two different synaptic structures. The stability of the prototypes is shown to increase when the fraction of neurons coding for the category goes down. Then the effect of synapse destruction on the retrieval is studied in two opposite situations : first analytically in sparsely connected networks, then numerically in completely connected ones. In both cases the behaviour of the structured network and that of the usual homogeneous networks are compared. When lesions increase, two transitions are shown to appear in the behaviour of the structured network when one of the patterns is presented to the network. After the first transition the network recognizes the category of the pattern but not the individual pattern. After the second transition the network recognizes nothing. These effects are similar to syndromes caused by lesions in the central visual system, namely prosopagnosia and agnosia. In both types of networks (structured or homogeneous) the stability of the prototype is greater than the stability of individual patterns, however the first transition, for completely connected networks, occurs only when the network is structured.
\end{abstract}

\section{Introduction.}

Attractor neural network (ANN) models [1] have been studied intensively since the pioneering work of J. Hopfield [2] and its subsequent analytical study [3]. In these models configurations or «patterns » coding for the memorized concepts are attractors of the dynamics of the network, and learning these configurations occurs via mechanisms of synaptic plasticity.

(*) Present address : INFN, Sezione di Roma, Istituto di Fisica, Università di Roma La Sapienza, P. le Aldo Moro, 2-00185 Roma, Italy. 
Following the original Hopfield model where all the memories are random and uncorrelated, several authors have studied networks where the stored information has a hierarchical structure [4]-[9]. In the simplest case of a one-level hierarchy, the stored patterns are grouped into categories. The prototypes or « ancestors » of the categories are uncorrelated, while individual patterns belonging to the same category are correlated with their « ancestor ». Other authors have considered structured networks with attractor dynamics $[10,11]$.

In this paper we study a model of ANN with functionally different groups or modules. We mostly concentrate on a simple model where one module codes for the category of the stored information, while the other module codes for the remaining information characterizing the individual patterns. One concrete example of such a network may be a system storing pictures of a set of objects in a common background. One module of the network may code for the particular object represented independently of its position, size or orientation, while the rest of the network codes for the remaining information in the picture.

The effect of lesions on the synaptic matrix on the behaviour of the network is then investigated, as in $[7,9]$. In particular, we consider the case when an individual pattern is presented to the network, after a certain amount of damage has been done to its synaptic structure. It is shown that the structured network, after some amount of damage has been done, does not stay in the vicinity of the attractor corresponding to the pattern but rather goes to a configuration close to the prototype, i.e. the network recalls the category of the pattern but not the individual information. This result is obtained with two different examples of synaptic matrices and in the two extreme cases of the completely connected (CC) and the sparsely connected (SC) network.

This behaviour (shift from individual to class recall) is similar to a syndrome of the central visual system called prosopagnosia and has been considered previously in the context of homogeneous attractor networks [7, 9]. M. Virasoro has showed [7] that in almost every (unstructured) network storing hierarchical patterns the neurons where the individual pattern is equal to their ancestor are stabler to noise than the neurons where they are different. However the simulations done on a particular $\mathrm{CC}$ homogeneous network show that the network never recalls the ancestor when an individual pattern is presented, though the ancestor is stabler to noise than the individual patterns. In this case the difference between these two stabilities does not seem strong enough to ensure then when the network is damaged, it flows to the attractor corresponding to the category when a pattern is presented. When similar simulations are done on a CC structured network, it exhibits for a wide range of parameters the prosopagnosia-like behaviour. The structure, in all cases considered, increases the stability of the prototypes. Furthermore the stability of the prototypes increases when the fraction of category-coding neurons decrease.

The organization of the paper is as follows : in section 2 the considered model is defined more precisely. In section 3 we determine the parameters useful for the study of the dynamics of the network. The probability distribution of stabilities of the structured network is calculated, first in the case of a particular synaptic matrix generalizing the work of reference [9], then for the maximally stable network. In the following section, we derive the one time step recursion relations for the overlaps of the network with the presented pattern and its ancestor. Then, in section 5, we study the effect of damage on the dynamics; here damage is modelled by random dilution on the synaptic matrix. First the case of the SC network is studied and the «phase diagrams » in the storage level/dilution plane are obtained for both synaptic matrices. Then the CC network is studied numerically, and we compare the results with the analytical results of the SC network, for both homogeneous and structured networks. In the last section we discuss our results. 


\section{The model.}

In this section we present a general formalism describing a structured ANN with an arbitrary number of modules. However in most of the paper we will consider a simple network with two modules.

The structured ANN is composed of $L$ groups $G_{k}(k=1, \ldots, L)$ of binary neurons. Group $G_{k}$ is composed of $N_{k}=b_{k} N$ neurons, and we have $\sum_{k} b_{k}=1$ so the total number of neurons is $N$. The stored information is coded in the synapses $\left\{J_{1 j}\right\}_{1, J}=1, \ldots$. At neuron $i$ the local field $h_{l}(t)$ at time $t$ is defined by

$$
h_{1}(t)=\sum_{j \neq 1} J_{i j} S_{j}(t)
$$

where $S_{j}(t)= \pm 1$ is the state of neuron $j$ at time $t$. The state of neuron $i$ at time $t+1$ is determined by

$$
S_{l}(t+1)=\operatorname{sign}\left(h_{l}(t)\right) \text {. }
$$

2.1 THE STORED INFORMATION. - The network stores a set of $p=\alpha N$ binary random patterns $\xi^{\mu}=\left\{\xi_{1}^{\mu}\right\}_{1=1, \ldots N}(\mu=1, \ldots, p) . \alpha$ defines the storage level of the network. The stored patterns are segmented in the different groups of neurons, each module coding for a different feature of the patterns. In each module $G_{k}$, a finite number $\nu_{k}$ of categories is coded. The categories are represented by binary words $\sigma_{h}^{\lambda}=\left\{\sigma_{i}^{\lambda}\right\}_{I=1, N_{k}}\left(\lambda=1, \ldots \quad \nu_{h}\right)$ called «prototypes» or « ancestors » of the class. In each site the prototypes are randomly and independently chosen +1 or -1 . If the pattern $\xi^{\mu}$ belongs to the category $\sigma_{k}^{\lambda}$ then for each site $i \in G_{k} \xi_{i}^{\mu}$ is drawn from the distribution

$$
P_{k}\left(\xi=\sigma_{i}^{\lambda}\right)=\frac{1+m_{k}}{2}, \quad P_{k}\left(\xi=-\sigma_{1}^{\lambda}\right)=\frac{1-m_{k}}{2}
$$

where $m_{k}$ is the bias of the patterns inside a class in group $G_{h}$. If the pattern $\xi^{\mu}$ is in category $\lambda$ we also use the notation $\xi^{\mu \lambda}$ Note that $\xi$ always denotes an individual pattern while $\sigma$ denotes a prototype.

In the following we will have to distinguish in each group in a pattern $\mu$ the sites $i$ where the pattern is equal to its prototype $\left(\xi_{1}^{\mu \lambda}=\sigma_{1}^{\lambda}\right)$ from those where they are different $\left(\xi_{1}^{\mu \lambda}=-\sigma_{l}^{\lambda}\right):$ an $\varepsilon$-site, or an $\varepsilon$-neuron, $(\varepsilon= \pm 1)$ indicates a neuron where $\xi_{1}^{\mu \lambda}=\varepsilon \sigma_{l}^{\lambda}$.

In the simplest case of a two-module network we concentrate on the case where one of the groups, $G_{1}=G_{\mathrm{C}}$ codes for the categories of the stored patterns, while they are uncorrelated in the remaining module $G_{2}=G_{\mathrm{D}}$, coding for details of the individual patterns independent from the category. In this two-module network we note $b_{1}=b, b_{2}=(1-b), m_{1}=m$ and $m_{2}=0$. When $b=1$ we recover the usual unstructured network storing patterns in hierarchical classes. When $b=0$ or $m=0$ the network stores uncorrelated patterns. In the following both networks will be referred to as «homogeneous".

Next we define parameters which are particularly useful for the study of the dynamics. We define

$$
\Delta_{l}^{\mu}=\frac{\xi_{i}^{\mu} \sum_{j} J_{i \jmath} \xi_{j}^{\mu}}{\sqrt{\sum_{l} J_{l j}^{2}}}
$$


as the local stability of pattern $\mu$ at neuron $i$. These parameters have the following properties : if for all sites $i \Delta_{l}^{\mu}>0$, then the pattern $\mu$ is a fixed point of the dynamics, and thus «memorized » by the network. Furthermore the larger the stabilities, the stabler to noise the patterns.

The stability parameter at site $i$ is defined by

$$
K_{\imath}=\min _{\mu=1, \quad p} \Delta_{1}^{\mu}
$$

We also define partial stabilities of the pattern $\mu$ at site $i$

$$
\Delta_{i k}^{\mu}=\frac{\xi_{i}^{\mu} \sum_{j \in G_{k}} J_{l j} \xi_{j}^{\mu}}{\sqrt{\sum_{j} J_{l j}^{2}}}
$$

for all groups $k$. These parameters reflect the influence of the different groups on the local stability of the pattern. We have for all $i$ and $\mu \Delta_{l}^{\mu}=\sum_{l} \Delta_{i k}^{\mu}$.

The stabilities of the prototypes are defined in a similar way. The local stability of ancestor $\lambda$ at site $i M_{1}^{\lambda}$ is

$$
M_{1}^{\lambda}=\frac{\sigma_{i}^{\lambda} \sum_{j} J_{i j} \sigma_{j}^{\lambda}}{\sqrt{\sum_{j} J_{i j}^{2}}}
$$

The importance of these parameters will appear more clearly when we will study the dynamics of the network.

2.2 THE SYNAPTIC STRUCTURE. - The network has a uniform connectivity $C$ : for each couple of neurons the probability that the connection between them is present is $C / N$. In the following we will consider two extreme cases :

- a completely connected (CC) network, where all neurons are connected to all the other neurons, i.e. $C=N$;

- a sparsely connected (SC) network, where $1 \ll C \ll \ln N$. In this network the dynamics can be solved completely, as shown in [12].

None of these cases is plausible biologically, however if we obtain similar results in these two extreme cases we may expect the behaviour of the network to be similar at any level of connectivity.

At every neuron the global strength of the incoming connections is the same, and we use the normalization (for all $i=1, \ldots, N$ )

$$
\sum_{j \neq i} J_{i j}^{2}=C
$$

We define, for each neuron $i$

$$
\gamma_{, k}=\frac{1}{C} \sum_{j \in G_{k}} J_{l j}^{2}
$$


These parameters measure the relative strength of the connections arriving at neuron $i$ from group $G_{k}$. If $\gamma_{1 k}=0$ no connections are present from $G_{k}$ to neuron $i$. We have, for all neurons $\sum_{k} \gamma_{1 h}=1$. In the following we suppose these parameters to be equal for neurons in the same group, i.e. for all neurons $i \in G_{\ell} \gamma_{1 k}=\gamma_{\ell k}$. The matrix $\left\{\gamma_{\ell k}\right\}_{\ell, h=1, \ldots N}$ defines the " architecture » of the structured network. In the case of the two-module network we define $\gamma_{1}=\gamma_{C}=\gamma_{11}$ and $\gamma_{2}=\gamma_{D}=\gamma_{21}$ which are the relative strengths of the connections coming from the category-coding group.

In the following two different synaptic matrices will be studied :

- First we use an explicit matrix which is a generalization of the Hebbian matrix used in [9]. The matrix is, for $i \in G_{k}$ and $j \in G_{\rho}$

$$
J_{i j}=\frac{U_{k} \delta_{k \ell}}{\sqrt{b_{k} \alpha C}} P_{i j}^{\ell}+\frac{\gamma_{h \ell}}{\sqrt{b_{k}\left(1-m_{h}^{2}\right)\left(1-m_{\ell}^{2}\right) \alpha C}}\left(Q_{1 j}^{k \ell}-\frac{c_{\ell}}{C} \sum_{j \in G_{\ell}} Q_{i j}^{k \ell} P_{l J}^{\ell}\right)
$$

where

$$
P_{l y}^{h}=\sum_{\lambda_{h}=1}^{n_{k}} \sigma_{l}^{\lambda_{h}} \sigma_{l}^{\lambda_{h}}
$$

and

$$
Q_{l j}^{\lambda \ell}=\sum_{\mu=1}^{p}\left(\xi_{l}^{\mu}-m_{\ell} \sigma_{l}^{\lambda_{k}(\mu)}\right)\left(\xi_{l}^{\mu}-m_{\ell} \sigma_{l}^{\lambda_{l}(\mu)}\right)
$$

where $\lambda_{k}(\mu)$ denotes the category to which pattern $\mu$ in group $G_{k}$ belongs. The first two terms in the r.h.s. of (7) are Hebb-like couplings for both classes and individuals. Note that the first term appears only for couplings connecting neurons inside the same group, as the categories differ from group to group. The third term has the same effect as in [9], i.e. it is useful in reducing noise in the stability parameters of the individual patterns, as we will see in the next section. The overall factors have been chosen so that the constraints (5) and (6) are satisfied. The parameters $c_{\ell}$ and $U_{k}$ measure the relative strengths of the first and third terms.

- Another approach is to study the space of all possible matrices that memorize a given set of patterns in order to derive their typical properties [7, 15]. For the sake of simplicity we only consider, for a given storage level, the matrix that optimizes the stability parameters at every site of the network. This network will be referred to as maximally stable (MS). Note that for the case of the homogeneous network it is shown in [9] that when the storage level goes to its critical value (i.e. maximal loading without errors) and when the bias inside categories goes to one the Hebbian matrix becomes the MS one. For an arbitrary set of patterns the Minover [13] or the Adatron [14] algorithms converge to the MS synaptic matrix.

\section{Stability parameters and distribution of local stabilities.}

In this section we determine for both Hebbian and MS networks the probability distribution of the stabilities of both individual patterns and prototypes. Our motivation is to make the detailed study of the dynamics possible. This will be done in the next section.

3.1 The HebBian NeTWORK. - We now concentrate on the synaptic matrix (7). In the thermodynamical limit $N \rightarrow \infty$ the partial stabilities $\Delta_{k \ell}$ at a $\varepsilon$-site in $G_{k}$ have Gaussian distributions $\rho^{F}\left(\Delta_{k \ell}\right)$ with mean values (for $\ell=1, ., L$ )

$$
\left\langle\Delta_{k \ell}\right\rangle_{\varepsilon}=\frac{1}{\sqrt{\alpha}}\left(\gamma_{k \ell}+\varepsilon m_{k}\left(U_{h} \delta_{k \ell}-\gamma_{k \ell}\right)\right) \text {. }
$$


The variance is independent of $\varepsilon$ and, for each group $G_{\ell}$, has a minimum for $c_{p}=1$ equal to

$$
\left\langle\Delta_{k \ell}^{2}\right\rangle_{\mathrm{c}}=\left\langle\Delta_{k \ell}^{2}\right\rangle_{f}-\left\langle\Delta_{k \ell}\right\rangle_{F}^{2}=\frac{\gamma_{k \ell}^{2}\left(1-m_{\ell}^{2}\right)}{b_{\ell}}
$$

Thus, from now on, we consider the matrix (7) with all $c_{\ell}=1$. The joint distribution $\rho^{\varepsilon}\left(\Delta_{k 1}, \ldots, \Delta_{k L}\right)$ of the partial stabilities at a $\varepsilon$-site is in this case the product of the Gaussian distributions $\rho^{\varepsilon}\left(\Delta_{k}\right)(\ell=1, \ldots, K)$, and thus the distribution of total stabilities in each group is also Gaussian. Its variance has a minimum for

$$
\gamma_{k \ell}=\frac{b_{\ell} v^{2}}{1-m_{\ell}^{2}}
$$

where

$$
\frac{1}{v^{2}}=\sum_{k} \frac{b_{k}}{1-m_{k}^{2}}
$$

From now on, we consider a network whose architecture is given by (10). The mean value of the stabilities in group $G_{k}$ is

$$
\left\langle\Delta_{k}\right\rangle_{E}=\frac{1}{\sqrt{\alpha}}\left(1+m_{k} \varepsilon\left(U_{h}-1\right)\right)
$$

and its variance is simply $\left\langle\Delta_{k}^{2}\right\rangle_{c}=v^{2}$. The stability of the ancestors does not fluctuate in the thermodynamical limit and is in group $G_{k} M_{k}=U_{k} / \sqrt{\alpha}$.

Now, in order to optimize the parameters $U_{k}$ in each group, we calculate the probability of error in a site $i \in G_{k}$ of the network, when its state is one of the stored patterns. This probability is given by the probability of having a negative stability parameter. When we minimize this probability we obtain the optimal $U$ parameters. They are given by

$$
U_{k}=1+\frac{\alpha v^{2}}{2 m_{k}} \ln \left(\frac{1+m_{k}}{1-m_{k}}\right) \text {. }
$$

Note that for a homogeneous network we recover the results of Reference [9].

In the two-module network, the optimal parameters are $\gamma_{C}=\gamma_{D}=b v^{2} /\left(1-m^{2}\right)$ where

$$
\frac{1}{v^{2}}=\frac{b}{1-m^{2}}+1-b=1+\frac{b m^{2}}{1-m^{2}} \text {. }
$$

The probability distribution of the stabilities in a $\xi$-site in $G_{\mathrm{C}}$ is

$$
\rho_{\mathrm{C}}^{\xi}(\Delta)=\frac{1}{v} G\left[\frac{\Delta}{v}-\frac{1}{\sqrt{\alpha v^{2}}}\left(1+\xi \frac{\alpha v^{2}}{2} \ln \left(\frac{1+m}{1-m}\right)\right)\right]
$$

where $G(x)=\exp \left(-x^{2} / 2\right) / \sqrt{2 \pi}$. In $G_{\mathrm{D}}$

$$
\rho_{\mathrm{D}}(\Delta)=\frac{1}{v} G\left(\frac{\Delta}{v}-\frac{1}{\sqrt{\alpha v^{2}}}\right) .
$$


The probability of error in $G_{\mathrm{C}}$ is thus

$$
\rho_{\mathrm{C}}=\sum_{\xi= \pm 1} \frac{1+\xi m}{2} H\left[\frac{1}{\sqrt{\alpha v^{2}}}\left(1+\xi \frac{\alpha v^{2}}{2} \ln \left(\frac{1+m}{1-m}\right)\right)\right]
$$

where

$$
H(x)=\int_{1}^{\infty} G(t) \mathrm{d} t
$$

and in $G_{\mathrm{D}}$ we have

$$
p_{\mathrm{D}}=H\left(\frac{1}{\sqrt{\alpha v^{2}}}\right)
$$

For every $b, m>0$ we have $p_{\mathrm{C}}<p_{\mathrm{D}}$. the error in the detail-coding group is always greater than in the category-coding group. Furthermore $p_{\mathrm{D}}$ increases with $b$ at fixed $m$ and increases with $m$ at fixed $b$, thus for all $b, m>0$

$$
p_{\mathrm{D}}<H\left(\frac{1}{\sqrt{\alpha}}\right) .
$$

Note that the r.h.s. is the probability of error in the Hopfield model (i.e. $b=0$, $m=0$ ). The storage capacity of the structured network will therefore be higher than the capacity of the usual Hopfield network, since for every neuron the probability of error is smaller than in a Hopfield network with the same storage level. When the bias in $G_{\mathrm{C}} m \rightarrow 1$ we have

$$
p_{\mathrm{D}} \sim \sqrt{\frac{\alpha(1-m)}{\pi b}} \exp \left(-\frac{b}{4 \alpha(1-m)}\right)
$$

thus $p_{\mathrm{D}}$ vanishes in the limit $m \rightarrow 1$, though in $G_{\mathrm{D}}$ patterns are uncorrelated.

3.2 SPACE OF COUPLINGS AND THE MS NETWORK. - In order to obtain the stability parameters of the MS structured network we proceed along the lines of [15]. For a neuron $i$ in $G_{\ell}$, and for a given storage level $\alpha$, we calculate the volume in the space of couplings of solutions of the inequalities (for $\mu=1, \ldots, p) \Delta_{i}^{\mu}>K_{\ell}$, subject to the global normalization condition (5). The optimal stability parameter $K_{\ell}^{\text {opt }}$ in group $G_{\ell}$ is then obtained when the volume of solutions vanishes so that no more couplings satisfy the inequalities. The fractional volume of solutions, i.e. the volume divided by the volume of the whole hypersphere (5), for a neuron $i$ in group $\ell$, is given by [15]

$$
V_{1}=\frac{\int \prod_{j} \mathrm{~d} J_{i j} \prod_{\mu} \Theta\left(\Delta_{i}^{\mu}-K_{\ell}\right) \delta\left(\sum J_{1 j}^{2}-N\right)}{\int \prod_{J} \mathrm{~d} J_{i j} \delta\left(\sum J_{i j}^{2}-N\right)}
$$

The typical fractional volume for all possible realizations of the set of patterns is obtained with the replica method. This requires the averaging of $\ln V$, over the probability distribution of the $\xi_{i}^{\mu}$ s. The typical volume is equal for neurons in the same group, since patterns are 
drawn from the same probability distribution at these sites. Thus the site index is replaced by a group index in the following, and we have for all $i \in G_{\ell} V_{1}=V_{\ell}$.

In Appendix A this fractional volume is calculated for the two-module network described in the last section. First we show the results for a fixed «architecture» (i.e. $\gamma_{1}$ and $\gamma_{2}$ are fixed), then we calculate the optimal architecture. Note again that algorithms like the minover [13] or the adatron [14] converge to couplings with the optimal "architecture ".

In the category coding group $G_{1}$ the optimal stability parameter $K_{1}$ and the stability of the ancestors $M$ is given for architecture $\left(\gamma_{1}, \gamma_{2}\right)$ and storage level $\alpha$ by the following equations

$$
\begin{gathered}
\alpha \sum_{\xi= \pm 1} \frac{1+m \xi}{2} \int_{\infty}^{\tilde{K}_{1}^{\xi}} \operatorname{Dt}\left(t-\tilde{K}_{\mathrm{l}}^{\xi}\right)^{2}=\zeta_{1} \\
\sum_{\xi= \pm 1} \xi \frac{1+m \xi}{2} \int_{\infty}^{\tilde{K}_{1}^{\xi}} \operatorname{Dt}\left(t-\tilde{K}_{\mathrm{l}}^{\xi}\right)=0
\end{gathered}
$$

where $\tilde{K}_{1}^{\xi}=\left(K_{1}-\xi m M\right) / \sqrt{1-\mu_{1}^{2}}$ and $D t$ is the Gaussian measure $D t=G(t) \mathrm{d} t$.

In the other group the stability parameter $K_{2}$ is given by

$$
\alpha \int_{\infty}^{\tilde{K}_{2}} \operatorname{Dt}\left(t-\tilde{K}_{2}\right)^{2}=\zeta_{2}
$$

with $\tilde{K}_{2}=K_{2} / \sqrt{1-\mu_{2}^{2}}$. In $(15,17) \mu_{k}=\sqrt{\gamma_{k} m^{2}}$, and the parameters $\zeta_{k}$ are (for $k=1,2)$

$$
\zeta_{k}=\frac{\left(\sqrt{b \gamma_{h}\left(1-m^{2}\right)}+\sqrt{(1-b)\left(1-\gamma_{h}\right)}\right)^{2}}{1-\mu_{h}^{2}}
$$

For every architecture $\zeta_{k}<1 ; \zeta_{k}=1$ if and only if

$$
\gamma_{h}=\frac{b v^{2}}{1-m^{2}}
$$

where $v$ is given by (13). Note that this is the architecture of the optimal Hebbian network.

To obtain the maximally stable (MS) network one has to optimize the stability parameters with respect to the architecture. This optimal architecture satisfies to $(k=1,2)$

$$
\alpha \sum_{\xi= \pm 1} P_{h}(\xi) \int_{\tilde{K}_{k}^{\xi}}^{\infty} D t\left(t-\tilde{K}_{h}^{\xi}\right)=\frac{\sqrt{\zeta_{k}}}{m^{2} K_{k}}\left(\sqrt{\frac{(1-b)\left(1-m^{2}\right)}{1-\gamma_{k}}}-\sqrt{\frac{b}{\gamma_{k}}}\right) .
$$

When $\alpha=0$, the optimal architecture is given by

$$
\gamma_{C}=\gamma_{D}=\frac{b\left(1-m^{2}\right)}{1-b m^{2}}
$$

In both groups the relative strength of the couplings coming from the category-coding group increases with the storage level and when $\alpha$ is such that in group $G_{k} K_{k}=0$ the parameter $\gamma_{k}$ is given by (19).

In figure 1 we show $K_{\mathrm{C}}$ and $K_{\mathrm{D}}$ versus the storage level $\alpha$ for $m=0.9$. Note that $K_{\mathrm{C}}$ is greater than the stability parameter of the MS homogeneous network with same bias. In 


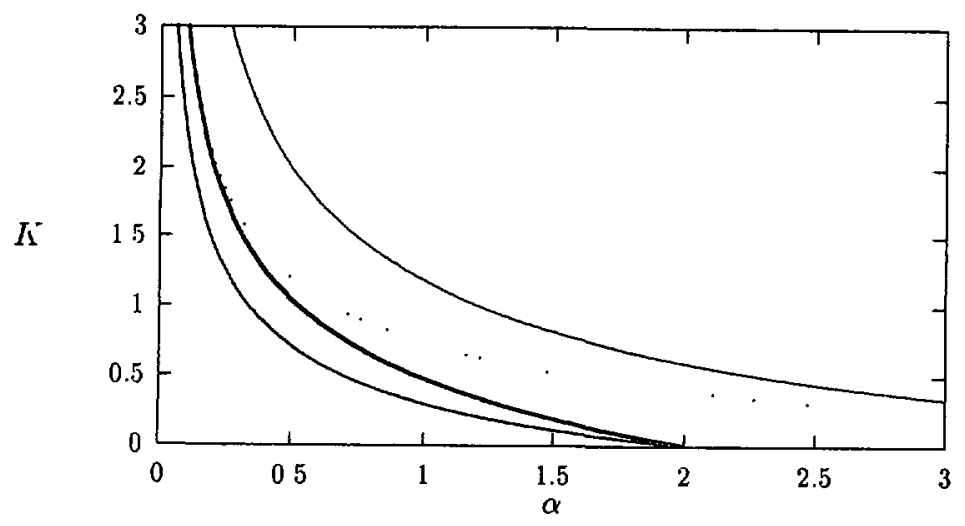

Fig. 1. - Stability parameters in the MS network versus the storage level. Bold curve : homogeneous network storing unbiased patterns $(b=0)$. Light curves: structured network with $b=0.5$ and $m=0.9$; higher curve : $K_{\mathrm{C}}$, lower curve : $K_{\mathrm{D}}$. Dotted curve : homogeneous network storing highly biased patterns $(b=1, m=0.9)$.

the detail-coding group $K_{\mathrm{D}}$ decreases with increasing $m$. This reflects the influence of the category-coding group : the greater the bias inside categories, the smaller the stabilities of the patterns in $G_{\mathrm{D}}$. In figure 2 we show the stability of the ancestors $M$ as a function of the storage level. It shows that the stability of the categories is thus also increased with respect to the homogeneous network with the same bias.

In figure 3 we show the variation of the stability parameters with the bias, for $\alpha=0.5$. Note that for $b<1$ the stability parameters of the category-coding group diverge in the limit $m \rightarrow 1$ while it stays finite in a homogeneous network $(b=1)$. Both $K_{\mathrm{C}}$ and $M$ increase when $b$ decreases.

Note that by calculating the optimal stability parameters we also determined the storage capacity $\alpha_{c}$ of this structured network: $\alpha_{c}$ is obtained when

$$
\min \left(K_{\mathrm{C}}, K_{\mathrm{D}}\right)=0 \text {. }
$$

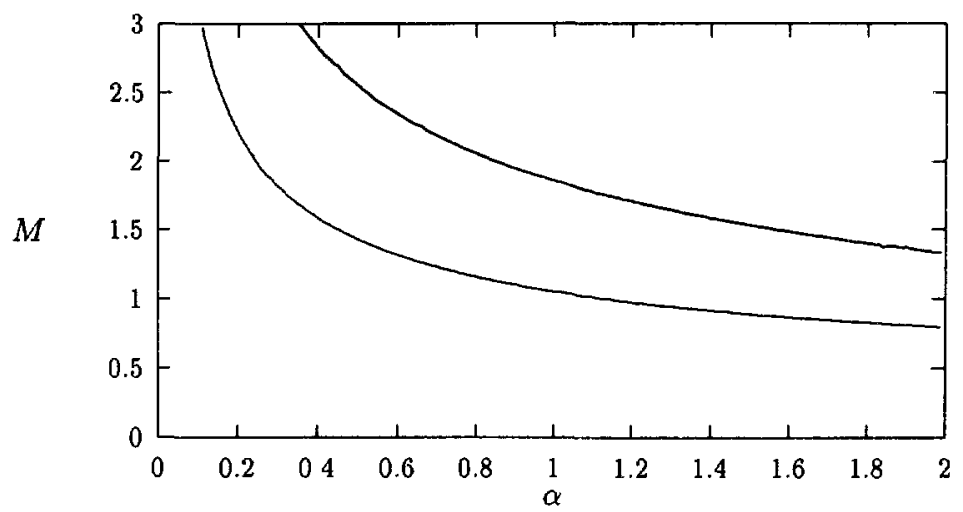

Fig. 2. - Stability of the ancestors $M$ in the MS network ver:sus the storage level. Bold curve : structured network with $b=0.5$ and $m=0.9$. Light curve: homogeneous network with $m=0.9$. 


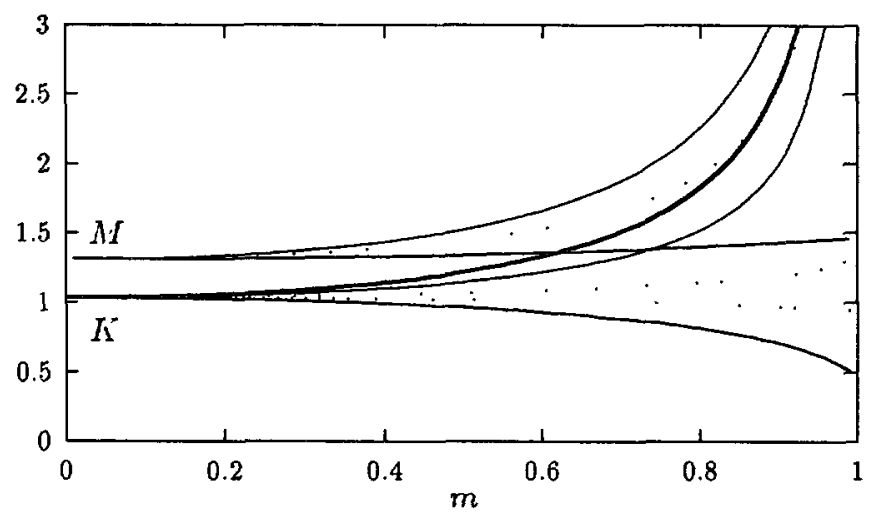

Fig. 3. - Stability parameters versus the bias $m$ in the MS network for $\alpha=0.5$. Curves for the stability of the ancestor $M:$ from the highest to the lowest curve; $b=0.1 ; b=0.5 ; b=1$. Curves for the stability parameters: from the highest to the lowest curve; $K_{\mathrm{C}}$ for $b=0.1 ; K_{\mathrm{C}}$ for $b=0.5$; $K$ for $b=1 ; K_{\mathrm{D}}$ for $b=0.5 ; K_{\mathrm{D}}$ for $b=0.1$.

Here we always have $K_{\mathrm{C}}>K_{\mathrm{D}}$ and when $K_{\mathrm{D}}=0$ we get $\alpha=2$. The storage capacity is therefore the same as in a homogeneous network storing unbiased patterns. This is not surprising since the patterns are uncorrelated in $G_{2}$.

In order to calculate the joint distributions of partial stabilities we proceed along the lines of [16]. These joint distributions are calculated in Appendix B. For the MS network we obtain, for $G_{1}$ and a $\xi$-site,

$$
\begin{aligned}
& \rho_{\mathrm{C}}^{\xi}\left(\Delta_{1}, \Delta_{2}\right)= \sqrt{\frac{2 \pi\left(1-\mu_{\mathrm{C}}^{2}\right)}{\gamma_{\mathrm{C}}\left(1-\gamma_{\mathrm{C}}\right)\left(1-m^{2}\right)}} G\left(\frac{\Delta_{1}-\xi m M}{\sqrt{\gamma_{\mathrm{C}}\left(1-m^{2}\right)}}\right) G\left(\frac{\Delta_{2}}{\sqrt{1-\gamma_{\mathrm{C}}}}\right) \times \\
& \times \exp \left(\frac{\left(\Delta_{1}+\Delta_{2}-\xi m M\right)^{2}}{2\left(1-\mu_{\mathrm{C}}^{2}\right)}\right) \rho^{\xi}\left(\Delta_{1}+\Delta_{2}, K_{\mathrm{C}}, \frac{M}{\sqrt{\gamma_{\mathrm{C}}}}, \mu_{\mathrm{C}}\right)
\end{aligned}
$$

where $\rho^{\xi}(\Delta, K, M, m)$ is the distribution function of local stabilities for a homogeneous MS network [17] with bias $m$ and stability parameters $K$ and $M$ :

$$
\rho^{\xi}(\Delta, K, M, m)=\frac{1}{\sqrt{1-m^{2}}} G\left(\frac{\Delta-\xi m M}{\sqrt{1-m^{2}}}\right) \Theta(\Delta-K)+H\left(-\frac{K-\xi m M}{\sqrt{1-m^{2}}}\right) \delta(\Delta-K) .
$$

For the detail-coding group $G_{2}$ the joint distribution yields

$$
\begin{aligned}
\rho_{\mathrm{D}}\left(\Delta_{1}, \Delta_{2}\right)=\sqrt{\frac{2 \pi\left(1-\mu_{\mathrm{D}}^{2}\right)}{\gamma_{\mathrm{D}}\left(1-\gamma_{\mathrm{D}}\right)\left(1-m^{2}\right)}} G\left(\frac{\Delta_{1}}{\sqrt{\gamma_{\mathrm{D}}\left(1-m^{2}\right)}}\right) G\left(\frac{\Delta_{2}}{\sqrt{1-\gamma_{\mathrm{D}}}}\right) \times \\
\\
\times \exp \left(\frac{\left(\Delta_{1}+\Delta_{2}\right)^{2}}{2\left(1-\mu_{\mathrm{D}}^{2}\right)}\right) \rho^{\xi}\left(\Delta_{1}+\Delta_{2}, K_{\mathrm{D}}, 0, \mu_{\mathrm{D}}\right)
\end{aligned}
$$

where in both expressions parameters $\gamma_{\mathrm{C}}, \gamma_{\mathrm{D}}, K_{\mathrm{C}}, M$ and $K_{\mathrm{D}}$ are given by equations (15-17, 
20 ). Note the similarity of these distributions to the case of an MS homogeneous network with bias $m[17]$.

Let us now consider how these distributions affect the dynamics of the network.

\section{One time step overlaps dynamics.}

4.1 DYNAMICS OF AN HOMOGENEOUS NETWORK. - We concentrate first on the simpler case of a homogeneous network. The dynamics of a network storing patterns organized in categories can be characterized by the overlaps of the network state, at time $t$, with the stored patterns

$$
q^{\mu}(t)=\frac{1}{N} \sum_{i=1}^{N} \xi_{t}^{\mu} S_{i}(t)
$$

and with the ancestors of the patterns

$$
a^{\lambda}(t)=\frac{1}{N} \sum_{t=1}^{N} \sigma_{i}^{\lambda} S_{i}(t) .
$$

For a network storing uncorrelated patterns, the time evolution of the overlaps is determined, to a large extent, by the distribution of the local stabilities $[16,17]$. For correlated patterns the time evolution also depends on the local stabilities of the ancestors of the patterns. At time $t$ the network has a finite overlap only with one ancestor ( $\lambda=1$ for example). Therefore we can describe the evolution of the network by $a(t)=a^{1}(t)$ and the new variables

$$
\tilde{q}^{\mu}(t)=\frac{q^{\mu}(t)-m a(t)}{1-m^{2}}
$$

for all patterns $\mu$ belonging to this category. Furthermore, we study the case where only one of these $\tilde{q}^{\mu}(t)$ is finite at time $t$. The evolution of the network is fully described by the variables $\tilde{q}(t)=\tilde{q}^{1}(t)$ and

$$
\tilde{a}(t)=\frac{a(t)-m q^{1}(t)}{1-m^{2}}
$$

The initial conditions in the network are thus $\tilde{q}(t)=O(1), \tilde{a}(t)=O(1), q^{\mu}(t)=m a(t)+$ $O(1 / \sqrt{N})$ for all other patterns in category $\lambda=1$, and for all other categories $a^{\lambda}(t)=O(1 / \sqrt{N})$. This means that the network has a significant overlap with only one pattern and its ancestor.

In this case, in the thermodynamical limit, the local fields $h_{l}(t)$ at time $t$ have a Gaussian distribution and are thus fully characterized by their mean value $E_{f}(t)$ (for a $\varepsilon$-neuron) and their variance $E_{2}(t)$. The overlaps at time $t+1$ after a single synchronous time-step of the network dynamics are related in the absence of noise to the overlaps at time $t, \tilde{q}(t)$ and $\tilde{a}(t)$, by

$$
\begin{gathered}
\tilde{q}(t+1)=\frac{1}{2} \sum_{\sigma} \int \mathrm{d} \Delta \rho_{\sigma}(\Delta) \operatorname{erf}\left(\frac{E_{\sigma}(t)}{\sqrt{2 E_{2}(t)}}\right) \\
\tilde{a}(t+1)=\frac{1}{2} \sum_{\sigma} \sigma \int \mathrm{d} \Delta \rho_{\sigma}(\Delta) \operatorname{erf}\left(\frac{E_{\sigma}(t)}{\sqrt{2 E_{2}(t)}}\right)
\end{gathered}
$$


where $\rho_{\varepsilon}(\Delta)$ is the distribution function of local stabilities for an $\varepsilon$-neuron. $E_{\sigma}(t)$ and $E_{2}(t)$ are given by

$$
\begin{gathered}
E_{\sigma}(t)=\Delta \tilde{q}(t)+\sigma M \tilde{a}(t) \\
E_{2}(t)=1-\left(\tilde{q}^{2}(t)+2 m \tilde{a}(t) \tilde{q}(t)+\tilde{a}^{2}(t)\right)
\end{gathered}
$$

where $M$ is the stability of the ancestor. Note that for $m=M=0$ we recover the recurrence relation of Reference [17].

In general these recurrence equations cannot be iterated because after one time step of the parallel dynamics the network states become correlated. However in the extreme case of the SC network the equations can be iterated till their fixed point. This will be done for the damaged network of section 5 .

4.2 StRUCTURED NETWORKS. - Let us now derive the one-time step recurrence equations in the case of a network with an arbitrary number of groups, each possibly having its own group of ancestors. In the case of a structured network, we need to consider overlaps in each group. The partial overlaps $q_{k}^{\mu}(t)$ at time $t$ of the network state with a given pattern $\mu$ restricted to neurons in the module $G_{k}$ are defined by

$$
q_{k}^{\mu}(t)=\frac{1}{N_{h}} \sum_{, \in G_{h}} \xi_{l}^{\mu} S_{l}(t)
$$

The total overlap of the network with the pattern $\mu$ is $q^{\mu}(t)=\sum_{k} b_{k} q_{k}^{\mu}(t)$. We define in a similar way the overlaps with the ancestors in each group

$$
a_{k}^{\lambda}(t)=\frac{1}{N_{h}} \sum_{i \in G_{k}} \sigma_{i}^{\lambda} S_{i}(t)
$$

We assume that at $t=0$ the network has in each group initial conditions similar to the last section, i.e. $a^{1}(t)=O(1), q_{k}^{1}(t)=m_{k} a^{1}(t)+O(1)$, and otherwise $q_{k}^{\mu}(t)=m_{k} a^{1}(t)+$ $O(1 / \sqrt{N})$ for patterns $\mu \neq 1$ in the category $\lambda=1$, and $a_{k}^{\lambda}(t)=O(1 / \sqrt{N})$ for all other categories, for all modules. This means the network has a significant overlap with only one pattern $(\mu=1)$ and its ancestors in each group $\left(\lambda_{k}(\mu)=1\right.$ for all $\left.k\right)$. Then, as in the last section, we define the renormalized overlaps (for $k=1, \ldots, L$ )

$$
\begin{aligned}
& \tilde{q}_{k}(t)=\frac{q_{h}^{1}(t)-m_{h} a_{k}^{1}(t)}{1-m_{h}^{2}} \\
& \tilde{a}_{k}(t)=\frac{a_{k}^{1}(t)-m_{h} q_{h}^{1}(t)}{1-m_{h}^{2}}
\end{aligned}
$$

The large $N$ limit implies as in the case of the homogeneous network that the local fields $h_{i}(t)$ are Gaussian random variables. The dynamics is thus characterized by its mean values $E_{\sigma}^{k}(t)$ (for a $\sigma$-neuron in group $k$ ) and its variance $E_{2}^{k}(t)$. Therefore we obtain the recursion relations for the noiseless dynamics $(k=1, ., L)$

$$
\tilde{q}_{k}(t+1)=\frac{1}{2} \sum_{v} \int d \boldsymbol{\Delta} \rho_{k}^{\sigma}(\boldsymbol{\Delta}) \operatorname{erf}\left(\frac{E_{\sigma}^{k}(t)}{\sqrt{2 E_{2}^{k}(t)}}\right)
$$




$$
\tilde{a}_{k}(t+1)=\frac{1}{2} \sum_{\sigma} \sigma \int d \Delta \rho_{k}^{\sigma}(\Delta) \operatorname{erf}\left(\frac{E_{\sigma}^{k}(t)}{\sqrt{2 E_{2}^{k}(t)}}\right)
$$

where $\rho_{k}^{\sigma}(\Delta)$ is the joint distribution of the partial stabilities in group $k$, for a $\sigma$-neuron. $E_{\varepsilon}^{k}$ and $E_{2}^{k}$ are given by

$$
\begin{gathered}
E_{\varepsilon}^{k}(t)=\sum_{\beta} \Delta_{l} \tilde{q}_{l}(t)+\varepsilon M_{k} \tilde{a}_{k}(t) \\
E_{2}^{k}(t)=1-\sum_{l \neq k} \gamma_{k \ell} \tilde{q}_{l}(t)^{2}-\gamma_{k \ell}\left(\tilde{q}_{k}(t)^{2}+2 m_{k} \tilde{a}_{k}(t) \tilde{q}_{k}(t)+\tilde{a}_{k}(t)^{2}\right) .
\end{gathered}
$$

In the SC network the coupled equations $(28,29)$ determine completely the dynamics and can be iterated to their fixed point.

Now we consider how the equations of the dynamics are affected if the network is perturbed.

\section{The effect of lesions on the dynamics.}

We now investigate the effect of damage on the dynamics of the network. In the following we concentrate on a particular type of damage, i.e. random dilution of the couplings. Other possible types of damage are reported in Appendix $\mathrm{C}$.

5.1 Homogeneous NeTwORKS. - First we study the effect of damage in a homogeneous network. The lesioned synaptic matrix is

$$
J_{i j}^{d}=d_{1 j} J_{i j}
$$

where the $d_{i j}$ are independent random variables taking the value 1 or 0 according to the probability distribution

$$
P\left(d_{\imath \jmath}=1\right)=d, \quad P\left(d_{\imath \jmath}=0\right)=1-d
$$

$d$ is thus the fraction of remaining links out of all previously present ones. In this situation because lesions on different sites are uncorrelated, the distribution of the local fields is still Gaussian. However its first two moments are now given by

$$
\begin{gathered}
E_{\sigma}(t)=d(\tilde{q}(t) \Delta+\sigma \tilde{a}(t) M) \\
E_{2}^{\sigma}(t)=d\left(1-d\left(\tilde{q}^{2}+2 m \tilde{q} \tilde{a}+\tilde{a}^{2}\right)\right) .
\end{gathered}
$$

For the SC network, given the degree of dilution, the initial conditions and the probability distribution of the stabilities, we can now completely study the dynamics. These dynamics are fully characterized by the fixed points of the coupled equations $(28,29)$ where the moments of the fields are given by $(36,37)$. We study these equations in the two specific cases discussed before, i.e. the Hebbian and MS networks. In both cases we start from an initial condition that has a finite overlap with one of the stored patterns $\tilde{q}(0)>0$ but $\tilde{a}(0)=0$. The fixed point overlaps are noted $q$ for the pattern and $a$ for its prototype.

When $m$ is positive, we observe in both cases three different types of fixed points:

- a fixed point with $q \gg a$. In this case the network recognizes the pattern (P fixed point);

- a fixed point with $a \gg q$ corresponding to the recognition of the category of the pattern (A fixed point) ; 
- the last fixed point has $q=a=0$ and corresponds to no recognition ( $\mathrm{N}$ fixed point). In the phase diagrams six different regions appear characterized by their stable fixed points : P, A, N, PA, AN and PAN. The notation indicates the stable fixed points in each region, and the order of appearance with decreasing $\tilde{q}(0)$. For example in the PA region the network recalls the pattern for a strong initial overlap and the ancestor otherwise.

5.1.1 The Hebbian network. - In this case, the distribution function is Gaussian and given by [9]

$$
\rho^{\xi}(\Delta)=\frac{1}{\sqrt{1-m^{2}}} G\left(\frac{\Delta-\xi m M-(1-m \xi) \alpha^{-1 / 2}}{\sqrt{1-m^{2}}}\right)
$$

where the stability of the ancestor $M$ is given by

$$
M=\frac{1}{\sqrt{\alpha}}+\frac{\sqrt{\alpha}\left(1-m^{2}\right)}{2 m} \ln \left(\frac{1+m}{1-m}\right) .
$$

For $m=0$ the recurrence relation for the overlap with one of the stored patterns simplifies to

$$
q(t+1)=\operatorname{erf}\left(\sqrt{\frac{d}{2 \alpha}} q(t)\right) .
$$

In this case the picture is the following : if $\alpha<2 d / \pi$, for every finite overlap $q(0)$ the network goes to the stored pattern. The fixed-point overlap is given by the following equation

$$
q=\operatorname{erf}\left(\sqrt{\frac{d}{2 \alpha} q}\right)
$$

If $\alpha>2 d / \pi$ the network does not recognise the pattern and the fixed point overlap is $q=0$, for every $q(0)$.

We show the phase diagram of the SC network storing highly biased patterns $(m=0.9)$ in figure 4. The main features of this diagram are as follows:

- Up to $\alpha \sim 1$ the network recognizes the pattern when the initial overlap is strong, at weak levels of dilution. When the dilution increases the behaviour of the network shifts from pattern recall to class recall. Then at high levels of dilution the network does not recognize anything.

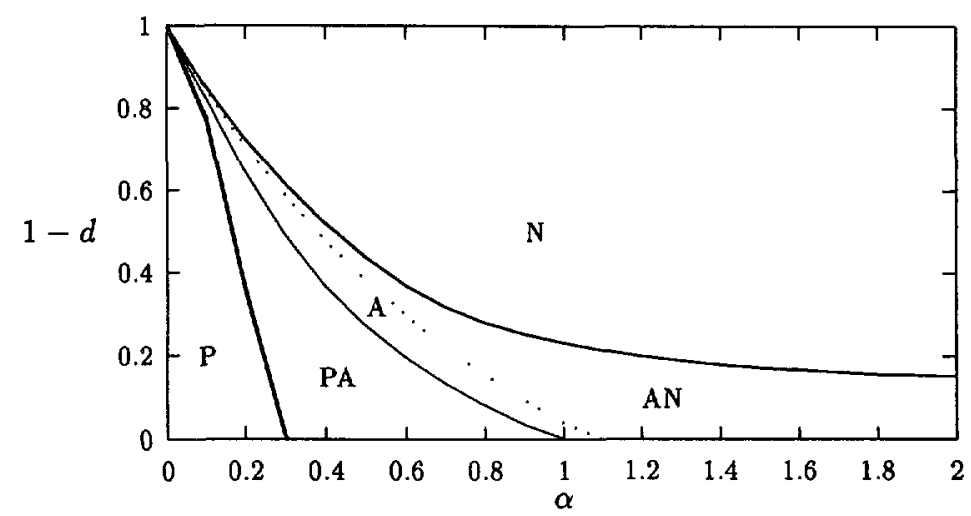

Fig. 4. - «Phase diagram » in $\alpha-d$ plane, for the homogeneous Hebbian network storing highly biased patterns $(m=0.9)$. 
- For high values of $\alpha$ the network still recognizes the category of the presented pattern when the initial overlap is strong, at weak levels of dilution.

5.1.2 The MS network. - The distribution functions of stabilities are given, for a $\xi$ site, by $[16,17]$

$$
\rho^{\xi}(\Delta)=\frac{1}{\sqrt{1-m^{2}}} G\left(\frac{\Delta-\xi m M}{\sqrt{1-m^{2}}}\right) \Theta(\Delta-K)+H\left(-\frac{K-\xi m M}{\sqrt{1-m^{2}}}\right) \delta(\Delta-K)
$$

where $K$ and $M$ are the stability parameters [15] for a given storage level $\alpha$. In figure 5 we show the "phase diagram " in the case of the MS network storing uncorrelated patterns $(m=0)$. This diagram is very similar to the diagram obtained in [19], showing that the effect of random dilution and temperature on the dynamics of the network are qualitatively similar.

The phase diagram of the MS network storing highly biased patterns $(m=0.9)$ is shown in figure 6. Note the similarity with the Hebbian network. This is not surprising as in [9] it is shown that in the limit $m \rightarrow 1$ both networks become identical. As expected the performance of the Hebbian network is better at a high degree of dilution for the recognition of both the category and the individual pattern. Furthermore even without dilution the basins of attraction of the ancestor are larger than in the MS case.

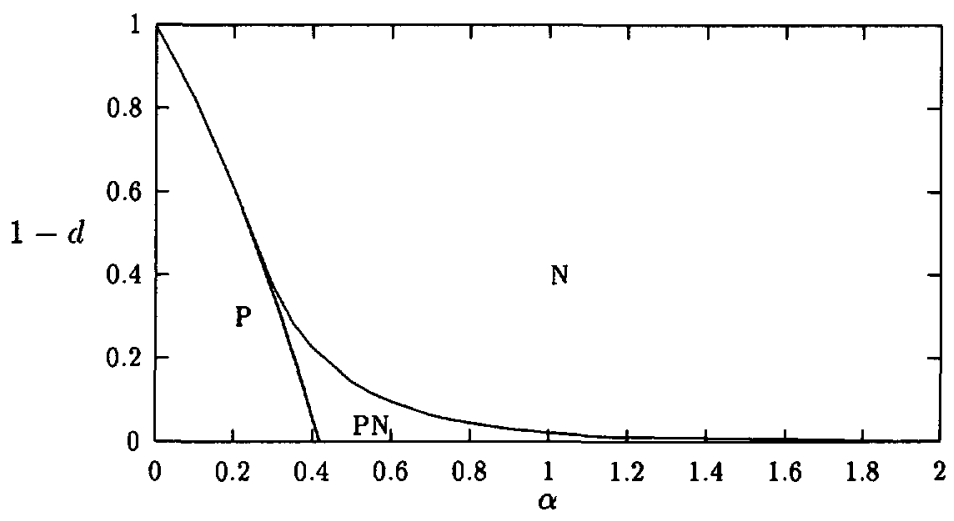

Fig. 5. - «Phase diagram" in $\alpha-d$ plane, for the homogeneous MS network with $m=0$ (no categories).

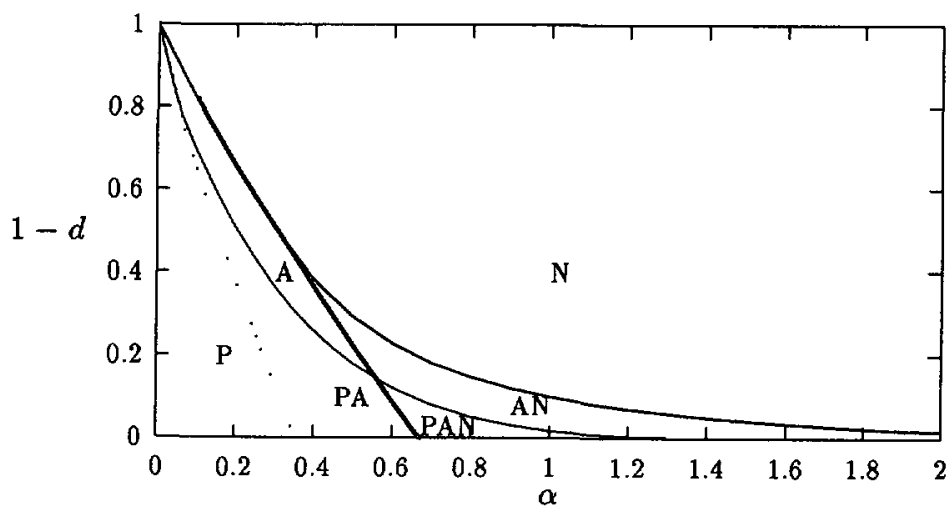

Fig. 6. - «Phase diagram » in $\alpha-d$ plane, for the homogeneous MS network with $m=0.9$. 
In figure 7 we show the fixed point overlaps $q$ and $a$ as a function of dilution for $\alpha=0.5$ and $\tilde{q}(0)=1$. It shows the two transitions that the system undergoes when dilution increases. At weak dilution levels the network recognizes well the presented pattern. After the first transition the network is not able to recognize the individual pattern, but the category is still well recognized. After the second transition the network does not recognise anything. Note that both transitions are discontinuous in the fixed point overlaps.

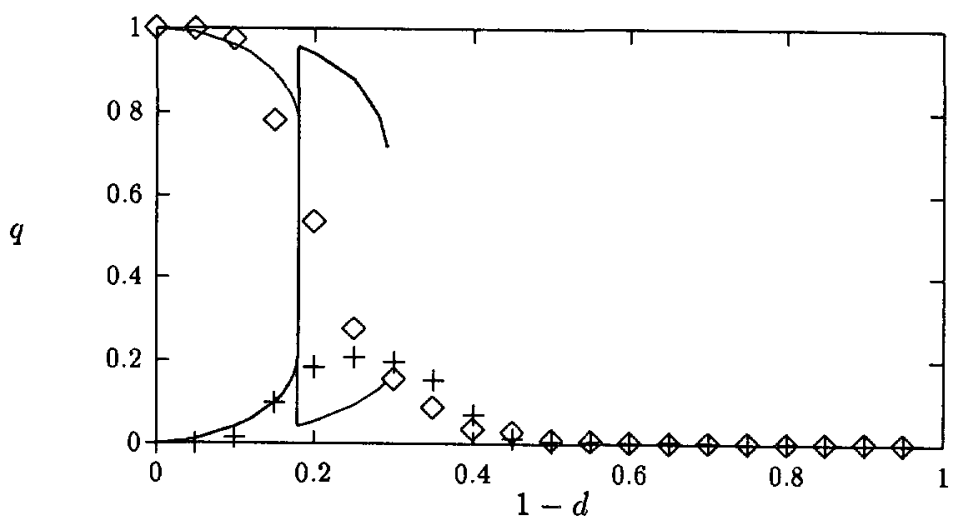

Fig. 7. - Fixed point overlaps $q$ and $a$ as a function of the dilution, for $\alpha=0.5, m=0.9$ and $\tilde{q}(0)=1$, in a MS network. Overlaps in the SC network : $a$ (bold curve) and $q$ (light curve). Overlaps in the CC network : $a(+)$ and $q(\diamond)$. The error bars, not shown for clarity, are of the order of 0.1 .

5.1.3 Numerical simulations on a CC network : the case of the MS network. - For the CC network, where the recurrence equations cannot be iterated, we use numerical simulations to study the dynamics. The simulated network has $N=200, p=100(\alpha=0.5), m=0.9$ and stores one category. The couplings are obtained with the Adatron algorithm [14] which converges to the MS network. To study the dynamics we proceed as follows. We start with an initial configuration equal to one of the stored patterns and we measure the fixed point overlaps $q$ and $a$. For each level of damage $d$, the results are averaged over 10 different sets of patterns and 100 patterns for each set. They are shown in figure 7 . We see that in this case only one transition occurs; the network never goes to the A fixed point. We do not know if this is due to a finite size effect or if the behaviour of the fully connected network is different than the SC one. However we will see in the following that for a structured MS network with the same characteristics ( $m=0.9, \alpha=0.5$ ) the two transitions observed analytically in the SC network coincide with those observed in numerical simulations on a $\mathrm{CC}$ network.

5.2 STRUCTURED NETWORKS. - For a structured network we model, as in a homogeneous network, the damage by random dilution. However, we now allow for different degrees of damage in the different groups. Examples of dissimetrical dilution are damage in only one module of neurons, or damage in connections between two specific groups.

The damaged synaptic matrix is given by (35) but now, if $i \in G_{k}$ and $j \in G_{\ell}$

$$
P\left(d_{i j}=1\right)=D_{k \ell}, \quad P\left(d_{i j}=0\right)=1-D_{k \ell}
$$

where $D_{k \ell}$ is the probability that the link between $i \in G_{k}$ and $j \in G_{p}$ has not been destroyed.

Since the randomness in the dilution is uncorrelated with that in the initial conditions, the local fields have again a Gaussian distribution and one gets new expressions for its first two 
moments

$$
E_{\sigma}^{k}(t)=\sum_{\ell} D_{k \ell} \Delta_{k \ell} \tilde{q}_{\ell}(t)+\sigma M_{k} \tilde{a}(t)
$$

and

$$
E_{2}^{k}(t)=\sum_{\ell \neq k} \gamma_{k \ell}\left(D_{k \ell}-D_{k \ell}^{2} \tilde{q}_{\ell}(t)^{2}\right)+\gamma_{k k}\left(D_{k k}-D_{k k}^{2}\left[\tilde{q}_{k}(t)^{2}+2 m_{h} \tilde{a}_{k}(t) \tilde{q}_{k}(t)+\tilde{a}_{k}(t)^{2}\right]\right) .
$$

Let us first consider two extreme cases, which correspond to two different physical situations. One is a uniform dilution : $D_{k \ell}=d$ for all $(k, \ell)$. It corresponds to a natural perturbation if the neurons are randomly distributed in space, and there is no relation between the spatial and the functional architecture. The second case is when neurons belonging to a same group are physically gathered in some module. One can consider in this case localized lesions. For example, if lesions affect module $k_{0}, D_{k \ell}=1$ for all $k, \ell \neq k_{0}$, and $D_{k k_{0}}=D_{k_{0} k}=d<1$ for all $k$.

In the following we consider the case of uniform lesion by random dilution in the simple two-module network. Different cases such as localized lesions will be considered in a future work.

One may wonder whether the results obtained with this particular choice of lesions, i.e. random dilution, are generic. We argue that any random and local perturbation of the couplings should lead to the same results; this is further discussed in Appendix C.

5.3 TwO-MODUlE NETWORK. - We now study how the dynamics of the simple two-group network is perturbed by lesions. Here the lesions are uniform so for all $k, \ell=1,2$ we have $D_{k \ell}=d$.

If the network has at time $t$ a macroscopic overlap with one pattern then the overlaps with the pattern in each group $q_{\mathrm{C}}(t)$ and $q_{\mathrm{D}}(t)$ and with the ancestor $a(t)$ are given by the recursion relations (note that, from now on we drop the tildes for the overlaps)

$$
\begin{gathered}
q_{\mathrm{C}}(t+1)=\frac{1}{2} \sum_{\sigma} \int d \Delta \rho_{\mathrm{C}}^{\sigma}(\Delta) \operatorname{erf}\left(\frac{E_{\sigma}^{\mathrm{C}}(t)}{\sqrt{2 E_{2}^{\mathrm{C}}(t)}}\right) \\
q_{\mathrm{D}}(t+1)=\int d \Delta \rho_{\mathrm{D}}(\Delta) \operatorname{erf}\left(\frac{E^{\mathrm{D}}(t)}{\sqrt{2 E_{2}^{\mathrm{D}}(t)}}\right)
\end{gathered}
$$

and

$$
a(t+1)=\frac{1}{2} \sum_{\sigma} \sigma \int d \Delta \rho_{\mathrm{C}}^{\sigma}(\Delta) \operatorname{erf}\left(\frac{E_{\sigma}^{\mathrm{C}}(t)}{\sqrt{2} E_{2}^{\mathrm{C}}(t)}\right)
$$

In the above equations the moments of the fields are given by

$$
E_{\sigma}^{\mathrm{C}}(t)=d[\Delta \mathbf{q}(t)+\sigma M a(t)], \quad E^{\mathrm{D}}(t)=d \Delta \mathbf{q}(t)
$$

and

$$
\begin{aligned}
E_{2}^{\mathrm{C}}(t) & =d-d^{2}\left[\gamma_{1}\left(q_{1}^{2}(t)+2 m a(t) q_{1}(t)+a^{2}(t)\right)+\left(1-\gamma_{1}\right) q_{2}^{2}(t)\right] \\
E_{2}^{\mathrm{D}}(t) & =d-d^{2}\left[\gamma_{2} q_{2}^{2}(t)+\left(1-\gamma_{2}\right)\left(q_{1}^{2}(t)+2 m a(t) q_{1}(t)+a^{2}(t)\right)\right]
\end{aligned}
$$


The fixed point overlaps are noted $q_{\mathrm{C}}, q_{\mathrm{D}}$ and $a$. The initial conditions are $q_{\mathrm{C}}(0)>0$, $q_{\mathrm{D}}(0)>0$ and $a(0)=0$. Again we study the MS and the Hebbian network. In both cases we choose $b=0.5$ and $m=0.9$, thus highly biased patterns in $G_{\mathrm{C}}$. The features of the phase diagrams are very similar to the homogeneous networks, however in both cases the regions where the ancestor of the pattern is retrieved is much larger. The individual patterns are thus less stable in the structured networks, while the basins of attraction of the ancestor get larger.

5.3.1 Two-module SC Hebbian network. - We show in figure 8 the phase diagram of the SC Hebbian network. Note the similarity with the homogeneous network. The region where the network retrieves the ancestor of the pattern has enlarged spectacularly.

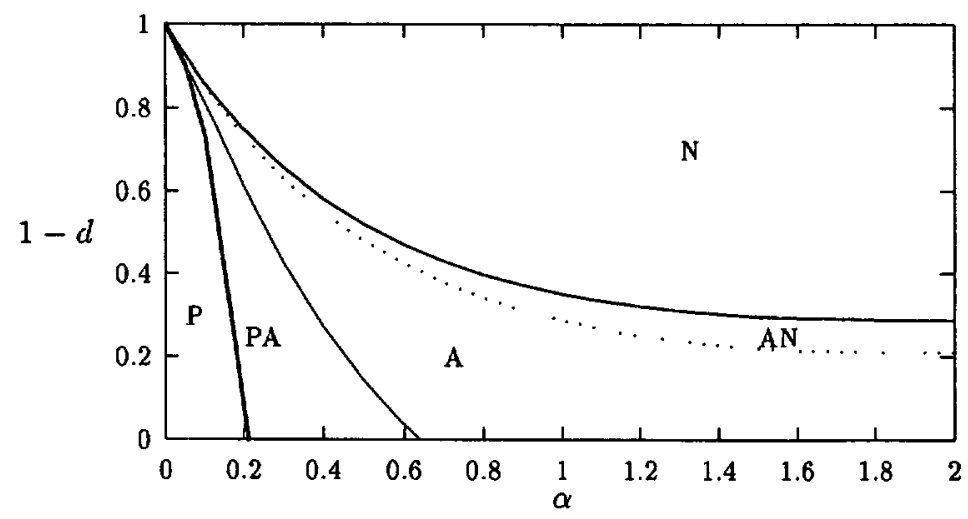

Fig. 8. - Phase diagram for the structured SC Hebbian network, for $b=0.5$ and $m=0.9$.

5.3.2 Two-module SC MS network. - The «phase diagram» is shown in figure 9. As expected, the results are very similar to the Hebbian network, as in the homogeneous networks. The region where the category is retrieved is much larger. Furthermore the structured Hebbian network shows the same robustness with respect to noise as the homogeneous Hebbian network, so that it surpasses the MS network at high dilution.

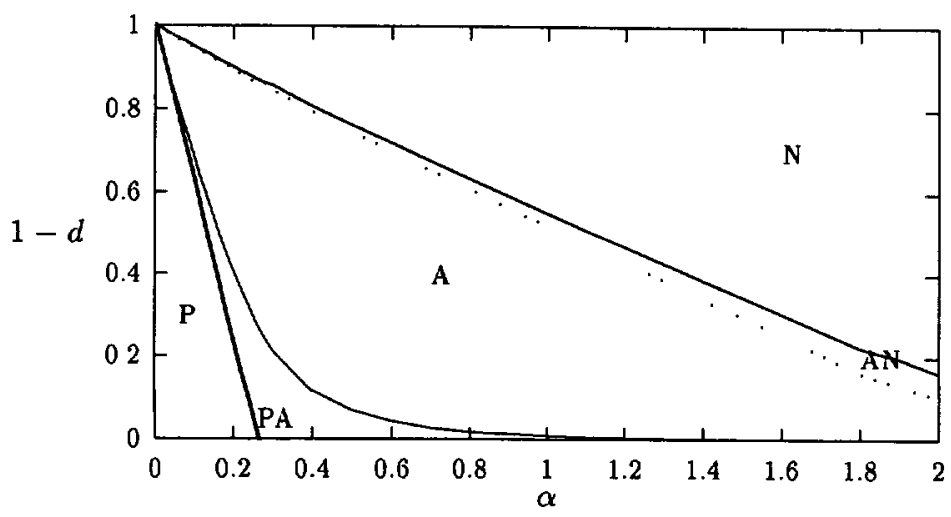

Fig. 9. - Phase diagram of the SC MS network, with $b=0.5$ and $m=0.9$. 
The fixed point overlaps are shown in figure 10 as a function of the dilution for $\alpha=0.5$. When the dilution is weak we observe a fixed point with $q_{\mathrm{C}}$ and $q_{\mathrm{D}}$ very near to one and $a=0$. In this case the network recognizes the presented pattern. When the dilution increases a first transition appears and the fixed point overlaps become $q_{1}=q_{2}=0$ and $a>0$ given by the equation

$$
a=\operatorname{erf}\left(\frac{d M a}{\sqrt{2 d\left(1-d a^{2}\right)}}\right)
$$

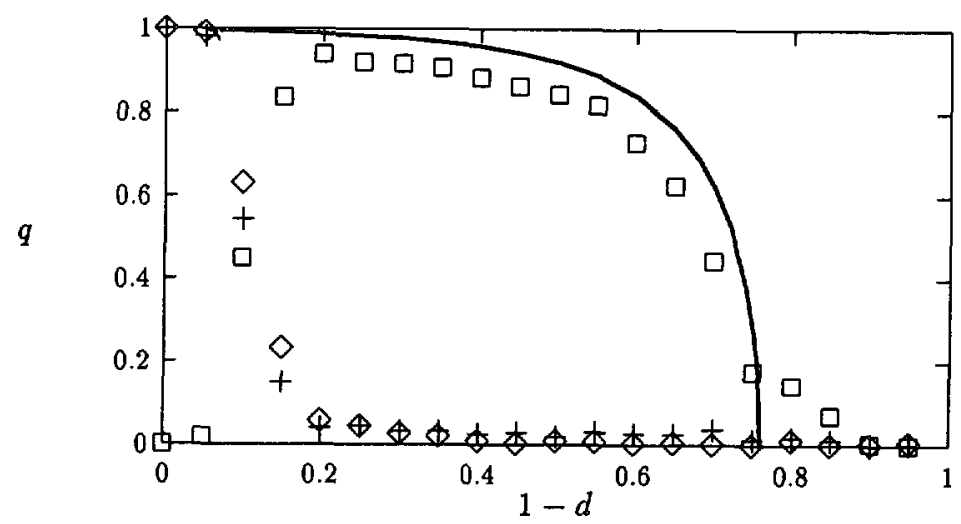

Fig. 10. - Fixed point overlaps in the two-group MS network, with $\alpha=0.5, b=0.5$ and $m=0.9$, as a function of the dilution parameter $1-d$. Overlaps in the SC network : $a$ (bold curve). $q_{\mathrm{C}}$ and $q_{\mathrm{D}}$ (Light curves, the curve for $q_{\mathrm{C}}$ is too close to one to be seen). Overlaps in the CC network : $a$ $(\square) ; q_{\mathrm{C}}(\diamond)$ and $q_{\mathrm{D}}(+)$. The error bars, as in figure 7 , are of the order of 0.1 .

In this case the network no longer recognizes the pattern but still the category to which it belongs. When the dilution is very strong a second transition happens and all the fixed point overlaps become zero; the network recognizes nothing. So the situation is qualitatively similar to the case of a homogeneous network. However the region where the category is retrieved is much larger in the case of the structured network. The other main difference is that the fixed points are no longer dissymetrical mixtures of the pattern and its ancestor but when the pattern is retrieved $a=0$ and when the ancestor is retrieved $q_{\mathrm{C}}=q_{\mathrm{D}}=0$.

5.3.3 Numerical simulations on the CC MS network. - Numerical simulations have been performed as in the last section in order to study the dynamics of the CC MS network; again we use $N=200$ and $p=100$. We choose $N_{1}=N_{2}=100$. Thus we have as in the SC network $\alpha=0.5, b=0.5$ and $m=0.9$. The couplings are calculated with the help of the Adatron algorithm and we present to the network one of the learned patterns $\left(\tilde{q}_{\mathrm{C}}(0)=1\right.$, $\tilde{q}_{\mathrm{D}}(0)=1$ and $a(0)=0$ ). Then we average the observed fixed point overlaps over 10 samples and 100 initial conditions for each sample. The results are given in figure 10 and show a remarkable similarity with the analytical calculation in the SC network. Here, contrary to the homogeneous network, the two transitions exist and their positions are very similar to the SC case. 


\section{Conclusions and outlook.}

We have investigated in this paper some properties of a structured ANN storing patterns organized in a hierarchical way. The focus has been put on a simple two-module model where one module codes for the category of the stored patterns while the second module codes for the remaining information. In all cases studied, the stability of the patterns is strengthened in the category-coding group, and increases with the correlation inside a class, as in an homogeneous network [15]. In the detail-coding group, the stability of the patterns decreases with increasing correlations inside the classes. Furthermore in this simple model the stability of the ancestor of the categories is always larger than in a homogeneous network with the same correlation inside the classes, and goes arbitrarily high when the correlation between the patterns increase. Another interesting fact is that it increases when the fraction of neurons in the category-coding group decreases.

Then we have studied the behaviour of this kind of networks with respect to noise. The problem of the effect of lesions on a network storing patterns organized in categories has already been studied by [7]. The fact that prototypes are stabler than individual patterns and that, for any pattern, the sites where it is common with its prototype are stabler than those where it differs from the prototype, has been interpreted as a shift from individual to class recall, and as such similar to a syndrome of the central visual cortex called prosopagnosia.

In the simple structured network studied we show a similar effect both analytically in a SC network and numerically in a fully connected network. After lesions have been done, the network, when an individual pattern is presented, goes to a configuration close to the prototype of the presented pattern. A remarkable agreement is obtained between the two opposite situations of the SC and the CC networks.

This behaviour does not appear for the simulated CC MS homogeneous network, though it appears in the corresponding SC one. Thus, though individual patterns are stabler in the sites that are common with the class prototype than in the other sites, the network, when an individual pattern is presented, never goes to the vicinity of the class prototype but rather flows away to a configuration uncorrelated to both pattern and prototype, when lesions are important enough. This could be a finite-size effect. Or the stability of the prototypes, though greater than the stability of the patterns themselves, may be too small in a homogeneous network to guarantee the shift from individual to class recall under lesion. In the SC homogeneous network, the region that exhibits prosopagnosia-like behaviour is much smaller than in the SC structured network, since the stability of the prototypes increases with the structure.

Note also that we have studied two particular synaptic matrices, which may have an untypical behaviour in the space of matrices storing this type of structured patterns.

Furthermore, these kinds of structured networks also allow us to investigate the possibility of localized lesions : these can be lesions inside a specific group or lesions inside connections between specific groups. They also allow the consideration of spatially organized networks where the connectivity is much stronger inside a group than between different groups. In this paper we have only considered homogeneous connectivity and lesions in an otherwise inhomogeneous network ; inhomogeneous connectivity and/or lesions will be considered in a future publication.

\section{Acknowledgements.}

I wish to thank Jean-Pierre Nadal and Hanoch Gutfreund whose ideas are at the origin of this paper, and Sylvio Franz for an enlightening discussion. I am grateful for the hospitality of INFN, Sezione di Roma where this work was completed. 


\section{Appendix A. Volume of solutions and optimal stabilities.}

We calculate, in a site $i$ of the network, the average $\left\langle\ln V_{1}\right\rangle$ over the quenched distribution of the patterns, where $V$, is defined in (14). The different parts of the network are treated separately and each of them is characterized by different order parameters. Here we consider a neuron $i$ belonging to the category-coding group $G_{1}$. The calculation is done using the replica method. We introduce $n$ replicas of the system, calculate $\left\langle V^{n}\right\rangle$, and then perform the usual analytical continuation to $n=0$. We define (for $k=1,2$ ) the order parameters (for $0 \leqslant a<b \leqslant n)$

$$
\begin{gathered}
q_{1 k}^{a b}=\frac{1}{\gamma_{1 k} N} \sum_{j \in G_{k}} J_{l j}^{a} J_{l j}^{b} \\
M^{a}=\frac{1}{\sqrt{N}} \sum_{j \in G_{l}} J_{l j}^{a}
\end{gathered}
$$

where $a$ and $b$ are replica indices. Calculation of the fractional volume with the replica symmetric ansatz $\left(q_{1 h}^{a b}=q_{1 h}\right.$ for all couples $(a, b)$ and $M^{a}=M$ for all replicas $\left.a\right)$ leads to the following saddle point equations for the order parameters

$$
-2 \alpha \sum_{\xi= \pm 1} \frac{1+\xi m}{2} \int D t \frac{\partial}{\partial \tilde{q}_{1}} \ln H\left(\tilde{X}_{1}^{\xi}\left(\tilde{q}_{1}, M, t\right)\right)=\frac{b_{k} q_{1 k}\left(1-\mu_{1}^{2}\right)}{\gamma_{1 k}\left(1-q_{1 k}\right)^{2}\left(1-m_{h}^{2}\right)}
$$

for $k=1,2$ and

$$
\sum_{\xi= \pm 1} \frac{1+\xi m}{2} \int D t \frac{\partial}{\partial M} \ln H\left(\tilde{X}_{1}^{\xi}\left(\tilde{q}_{1}, M, t\right)\right)=0
$$

where

$$
\begin{gathered}
D t=\frac{1}{2 \pi} \exp \left(-\frac{t^{2}}{2}\right) \mathrm{d} t \\
H(x)=\int_{1}^{\infty} D z \\
\tilde{X}_{1}^{\xi}\left(\tilde{q}_{1}, M, t\right)=\frac{1}{\sqrt{1-\tilde{q}_{1}}}\left(\frac{K_{1}-\xi M}{\sqrt{1-\mu_{1}^{2}}}+\sqrt{\tilde{q}_{1} t}\right) \\
\tilde{q}_{1}=\frac{\sum_{k} \gamma_{1 k} q_{1 k}\left(1-m_{k}^{2}\right)}{1-\mu_{1}^{2}}
\end{gathered}
$$

and $\mu_{1}^{2}=\gamma_{11} m^{2}$.

The left hand side of equations (48), (49) still involves the single site average over $\xi$ which depends on the site index $i$ in view of equation (1). For each site there is one saddle point equation (48) for every value of $k$ but since the left hand side does not depend on $k$ the expression on the right hand side is also $k$ independent. The fractional volume then vanishes when all $q_{1 /}$ s go to one. Because of equation (50) $\tilde{q}_{1}$ also goes to one. This limit has to be taken with the constraint that the right hand sides in (48) are equal for all $k$. When this is done one obtains equations (15), (16). For the detail-coding group the 
calculation is similar, but does not involve any parameter $M$, as no ancestor exists in this group. The stability parameter is in this case given by equation (17).

\section{Appendix B. Distribution of local stabilities.}

In the $\ell$ group the typical distributions $\rho_{\ell}^{\sigma}(\boldsymbol{\Delta})$ is given by

$$
\rho_{\ell}^{\sigma}(\Delta)=\left\langle\prod_{k} \delta\left(\Delta_{k}-\left(\Delta_{k}\right)_{l}^{\mu}\right)\right\rangle_{\mathrm{J}, \Xi}
$$

where we average over the probability distribution of the patterns and over all couplings that memorize these patterns. These typical joint distributions can be calculated using the same kind of techniques as in the last section. In the following we proceed along the lines of [16] and derive the typical distributions below and at criticality.

B.1 JOINT DISTRIBUTIONS FOR NETWORKS IN GARDNER'S SPACE. - The typical joint distribution function for a network in the volume defined in section 3 is given, in group $G_{p}$, by

$$
\rho_{\ell}(\mathbf{\Delta})=\int \prod_{\ell=1}^{2} D t_{k} \Psi \xi(\Delta, \mathbf{t}) \Theta\left(\Delta-K_{\ell}\right)
$$

with

$$
\begin{aligned}
& \Psi_{\xi}^{\xi}(\mathbf{\Delta}, \mathbf{t})= \\
& \frac{\prod_{k} \sqrt{\frac{2 \pi}{\left(1-m_{k}^{2}\right) \gamma_{\ell_{k}}\left(1-q_{\ell_{k}}\right)}} \exp \left(-\frac{1}{2} \frac{\left(\Delta_{k}+m_{k} M_{\ell h} \xi+t_{k} \sqrt{\left.\left(1-m_{k}^{2}\right) \gamma_{\ell_{k} q_{k}}\right)^{2}}\right.}{\left(1-m_{k}^{2}\right) \gamma_{\ell_{k}}\left(1-q_{\ell_{k}}\right)}\right)}{H\left(\frac{K_{\ell}+\sum_{k} \xi m_{k} M_{\ell_{k}}+t_{l} \sqrt{\left(1-m_{k}^{2}\right) \gamma_{\ell_{k}} q_{\ell_{k}}}}{\sqrt{\sum_{k=1}^{L}\left(1-m_{k}^{2}\right) \gamma_{\ell_{k}}\left(1-q_{\ell_{k}}\right)}}\right)}
\end{aligned}
$$

In the above equations the order parameters $M_{\ell_{k}}=M \delta_{k\}} \delta_{\ell_{1}}$ and $q_{\ell_{k}}$ are given by the saddle point equations (48) and (49).

B. 2 JOINT DISTRIBUTIONS FOR THE MS NETWORK. - We consider the joint distribution in the group $\ell$. When the stability parameters go to their critical value all the $q_{\ell_{k}}$ go to 1 , and the parameters $K_{\ell}$ and $M_{\ell}$ are determined by (15) and (16). In this limit the joint distributions can be expressed with a Gaussian part $g_{\ell}^{\sigma}(\Delta)$ and a function $\phi_{\ell}^{\sigma}$ depending only of the sum of the partial stabilities $\Delta=\sum_{k} \Delta_{k}$; we have

$$
\rho_{\ell}^{\sigma}(\Delta)=g_{\ell}^{\sigma}(\Delta) \phi_{\ell}^{\sigma}(\Delta)
$$

and

$$
\begin{aligned}
& g_{\xi}^{\xi}(\Delta)=\prod_{k=1}^{L} \sqrt{\frac{1}{2 \pi\left(1-m_{k}^{2}\right) \gamma_{\ell_{h}}} \exp \left(-\frac{1}{2\left(1-m_{h}^{2}\right) \gamma_{\ell_{k}}}\left(\Delta_{h}-\xi m_{\ell} M_{\ell h}\right)^{2}\right)} \\
& \phi_{\ell}^{\xi}(\Delta)=\Theta\left(\Delta-K_{\ell}\right)+\sqrt{2 \pi\left(1-\mu_{\ell}^{2}\right)} \exp \left(\frac{\left(\tilde{K}_{\ell}^{\xi}\right)^{2}}{2}\right) H\left(-\tilde{K}_{\ell}^{\xi}\right) \delta\left(\Delta-K_{\ell}\right) .
\end{aligned}
$$


Appendix C. Other possible types of noise on the synaptic matrix : the example of static synaptic noise.

Here we consider the simple case of a static synaptic noise :

$$
J_{1 \jmath}^{s}=J_{\imath \jmath}+\eta_{\imath \jmath}
$$

The $\eta_{1}$ are independent Gaussian random variables such that, if neurons $i$ and $j$ belong to groups $k$ and $\ell\left\langle\eta_{1 j}\right\rangle=0$ and

$$
\left\langle\eta_{, j} \eta_{\prime^{\prime} \jmath^{\prime}}\right\rangle=\frac{E_{\ell \ell}^{2}}{\sqrt{N}} \delta_{u^{\prime}} \delta_{\jmath^{\prime}} .
$$

The noise is uniform for synapses connecting one given group to another given group, but may change from one group to another. A straightforward calculation gives that this system is exactly equivalent to the diluted network with the probabilities of dilution given by

$$
D_{k \ell}=d_{k} \equiv \frac{1}{1+\sum_{\ell} b_{\ell} E_{k \ell}^{2}}
$$

More generally, one may expect that similar results will hold for any local perturbation (where for a given function $f$ each coupling $J_{i j}$ is replaced by $f\left(J_{i j}\right)$ ), as is the case for the standard Hopfield model [20].

\section{References}

[1] Amit D. J., Modeling Brain Function (Cambridge University Press, Cambridge, 1989).

[2] Hopfield J. J., Proc. Natl. Acad. Sci. U.S.A. 79 (1982) 2554.

[3] Amit D. J., Gutfreund H., Sompolinsky H., Phys. Rev. Lett. 55 (1985) 1530.

[4] Parga N., Virasoro M. A., J. Phys. France 47 (1986) 1857.

[5] Dotsenko V., Physica 140A (1986) 410.

[6] Feigelman M. V., Ioffe L. B., Int. J. Mod. Phys. B 1 (1987) 51.

[7] Virasoro M. A., Europhys. Lett. 7 (1988) 293.

[8] Gutfreund H., Phys. Rev. A 37 (1988) 570.

[9] Franz S., Amit D. J., Virasoro M. A., J. Phys. France 51 (1990) 387.

[10] Fassnacht C., Zippelius A., Networks 2 (1991) 63.

[11] Krey U., Poppel G., Z. Phys. B 76 (1989) 513.

[12] Derrida B., Gardner E., Zippelius A., Europhys. Lett. 4 (1987) 167.

[13] Krauth W., Mezard M., J. Phys. A : Math. Gen. 20 (1987) L745.

[14] Anlauf J. K., Biehl M., Europhys. Lett. 10 (1989) 687.

[15] Gardner E., J. Phys. A : Math. Gen. 21 (1988) 257.

[16] Kepler T. B., Abbott L. F., J. Phys. France 49 (1988) 1657.

[17] Krauth W., Mezard M., Nadal J. P., Complex Syst. 2 (1988) 387.

[18] Domany E., Kinzel W., Meir R., J. Phys. A : Math. Gen. 22 (1989) 2081.

[19] Amit D. J., Horner H., Evans M., Wong K. Y. M., J. Phys. A: Math. Gen. 23 (1990) 3361.

[20] Sompolinsky H., Heidelberg Colloquium on Glassy Dynamics, Lecture Notes in Physics, J. L. Van Hemmen, I. Morgenstern Eds. (Springer, Berlin, 1987) p. 275. 